Gut, 1973, 14, 89-93

\title{
Lymphocyte transformation in response to phyto- haemagglutinin in primary biliary cirrhosis: The search for a plasma inhibitory factor
}

\author{
R. A. FOX, F. J. DUdley, M. SAMUEls, J. Milligan, AND \\ SHEILA SHERLOCK ${ }^{1}$
}

From the Department of Medicine, Royal Free Hospital, London

SUMMARY The aim of this study was to determine the importance of plasma inhibitory factors in producing the impaired phytohaemagglutinin (PHA)-induced lymphocyte transformation that is seen in some patients with primary biliary cirrhosis. Twenty-six normal subjects and 12 patients with primary biliary cirrhosis were studied. The lymphocytes from each subject were cultured in the presence of autologous plasma and also in the presence of homologous plasma from a normal subject or from a patient with primary biliary cirrhosis. The results confirm that a proportion of patients with primary biliary cirrhosis are anergic and that the impaired lymphocyte transformation to PHA in vitro can be partially accounted for by the presence of inhibitory factors in the plasma. However, these factors are unlikely to account completely for the impaired transformation, it being probable that there are also abnormalities of the $T$ lymphocytes themselves.

The efficacy of phytohaemagglutinin (PHA) in stimulating the blast transformation of T-lymphocytes depends upon a number of factors, for example, the use of a suitable quantity of an active preparation of PHA and the presence of a population of lymphocytes capable of responding. Other factors need to be taken into account when assessing the response of the lymphocytes and it is well recognized that in certain situations inhibitory factors in the plasma can block the action of PHA. Plasma inhibitory factors resulting in impaired lymphocyte transformation to PHA have been found in alcoholic cirrhosis (Hsu and Leevy, 1971), in syphilis (Levene, Turk, Wright, and Grimble, 1969), in cirrhosis and coeliac disease (Winter, McCarthy, Reed, and Yoffey, 1967), and in other situations. The exact nature and mechanism of action of these inhibitory factors remains to be defined.

In vitro lymphocyte transformation to PHA has been investigated in patients with primary biliary cirrhosis (Fox, James, Scheuer, Sharma, and Sherlock, 1969; Fox, 1971). A significant proportion of patients with primary biliary cirrhosis were shown to have impaired transformation. In these studies the lympho-

${ }^{1}$ Requests for reprints should be addressed to S.S., Department of Medicine, Royal Free Hospital, Gray's Inn Road, London, WC1X 8LF.

Received for publication 28 November 1972. cytes and the other white cells were separated from the peripheral blood and resuspended in autologous plasma. The lymphocytes were not separated from other cells and were not washed. Although impaired transformation was more likely to be found in advanced disease and was not found in the very early cases, there was no apparent correlation with any index of liver function and the cause of the impairment was not clear. Impaired transformation to PHA was also found to correlate with other tests of the functional capacity of $T$ lymphocytes-there was an increased incidence of both negative tuberculin tests and failure to be sensitized to dinitrochlorobenzene in the patients with primary biliary cirrhosis compared with control subjects. In view of the granulomatous nature of primary biliary cirrhosis the finding of impaired $T$ lymphocyte function in such patients is of great interest, although it must be pointed out that such impairment was more frequently found at the stage of the disease when the granulomas have disappeared.

The reason for the anergy remains elusive however. Although it is concluded that it occurs with advanced disease, it is not an inevitable occurrence. There is no correlation between patients which allows one to predict whether impaired lymphocyte responsiveness is likely to be found or not.

In view of these findings it was decided to perform 
a series of experiments to determine if there were plasma factors inhibiting lymphocyte transformation to PHA in patients with primary biliary cirrhosis.

\section{Subjects}

Two sets of experiments were performed. The first set consisted of seven experiments in which blood was collected from 14 healthy volunteers. There were 12 males and two females and the ages ranged from 26 to 36. There was no evidence of disease in any subject and most were laboratory and hospital personnel.

In the second set of experiments blood was removed from 12 more healthy volunteers and 12 patients with primary biliary cirrhosis. In each experiment on a patient transformation was simultaneously performed on a healthy subject with presumed normal transformation. All 12 patients were diagnosed as having primary biliary cirrhosis. The diagnosis was reached by taking the histological appearances of the liver in conjunction with the clinical picture. The mitochondrial antibody test was positive in all patients and there were 10 females and two males.

\section{Method}

The method used was a modification of that of Crowther, Fairley, and Sewell (1969). Fifty ml venous blood was withdrawn into plastic syringes containing 500 units heparin (preservative free). The blood was well mixed and discharged into sterile containers, with $100 \mathrm{mg}$ finely divided carbonyl iron powder (type SF, Fine Dyestuffs and Chemicals Ltd). The containers were then rotated on a Matburn cell mixer at $25 \mathrm{rpm}$ at $37^{\circ} \mathrm{C}$ for 30 minutes. At the end of this time $1 \mathrm{ml}$ of $1 \%$ methyl cellulose was added to each $10 \mathrm{ml}$ of blood. The containers were left to stand upright at $37^{\circ} \mathrm{C}$ for 30 minutes, and at the end of this time the supernatant was drawn off. The red cells, together with the phagocytic white cells which had ingested the iron, were left behind having sedimented at a faster rate than the lymphocytes.

The lymphocytes were then washed three times in tissue culture solution (TC 199 Wellcome Labs) at $37^{\circ} \mathrm{C}$. After the final washing the lymphocytes were resuspended in tissue culture fluid to a fifth of the original volume of plasma. An aliquot was removed and total and differential white cell counts were performed. Eighty to ninety-eight percent of the final white cell population consisted of small lymphocytes.

The final culture volume in each tube was $3 \mathrm{ml}$ which contained 2 million lymphocytes. The appro- priate volume of lymphocyte suspension was added to each tube and the volume made up to $3 \mathrm{ml}$ by the addition of tissue culture medium and plasma in the ratio $4: 1$.

All cultures were run in triplicate and in each experiment the lymphocytes from two subjects (normal and normal or normal and patient) of the same major blood groups were cultured. The lymphocytes from each subject were cultured in the presence of autologous plasma and also in the presence of homologous plasma from a normal subject or a patient with primary tiliary cirrhosis. Each type of culture was performed in both the presence and absence of PHA.

The tubes were cultured at $37^{\circ} \mathrm{C}$ for 72 hours and at the beginning of the culture period $0.05 \mathrm{ml}$ PHA-P (Difco) was added to each of the appropriate tubes. Twenty hours before stopping the culture, by the addition of ice-cold normal saline, $0.25 \mu \mathrm{Ci}$ of tritiated thymidine was added to each tube.

The tubes were prepared for liquid scintillation counting after the cells had been washed three times in ice-cold saline. The method used has been described (Fox et al, 1969). The results are expressed as counts per minute per million lymphocytes.

\section{Results}

There were seven experiments involving 14 normal subjects whose lymphocytes were stimulated in their own and in another normal plasma and 12 experiments with 12 patients and 12 normals.

The transformation of unstimulated control lymphocytes varied little-mean 705 counts per minute per million lymphocytes (c/min/m lymphocytes) with a range from 106 to $1735 \mathrm{c} / \mathrm{min} / \mathrm{m}$ lymphocytes.

The normal response to PHA in the 26 normal control subjects in autologous plasma was quite wide: $12996 \mathrm{c} / \mathrm{min} / \mathrm{m}$ lymphocytes to $102737 \mathrm{c} / \mathrm{min} / \mathrm{m}$ lymphocytes, mean 43249 (Fig. 1). The PHA-induced transformation of lymphocytes from 12 patients with primary biliary cirrhosis when cultured in the presence of their own plasma are also seen in Figure 1. Five of the 12 patients had impaired transformation with counts below $50 \%$ of the lower limit of normal. The transformation in the remaining seven patients was within the normal range.

The results of the seven experiments on 14 normal subjects are shown in Figure 2. Lymphocyte transformation to PHA in homologous plasma from a healthy individual was within the normal range. However, changing the plasma resulted in a change in the transformation on each occasion. Seven showed an increase and seven a decrease. It is concluded that there is no constant effect produced by 


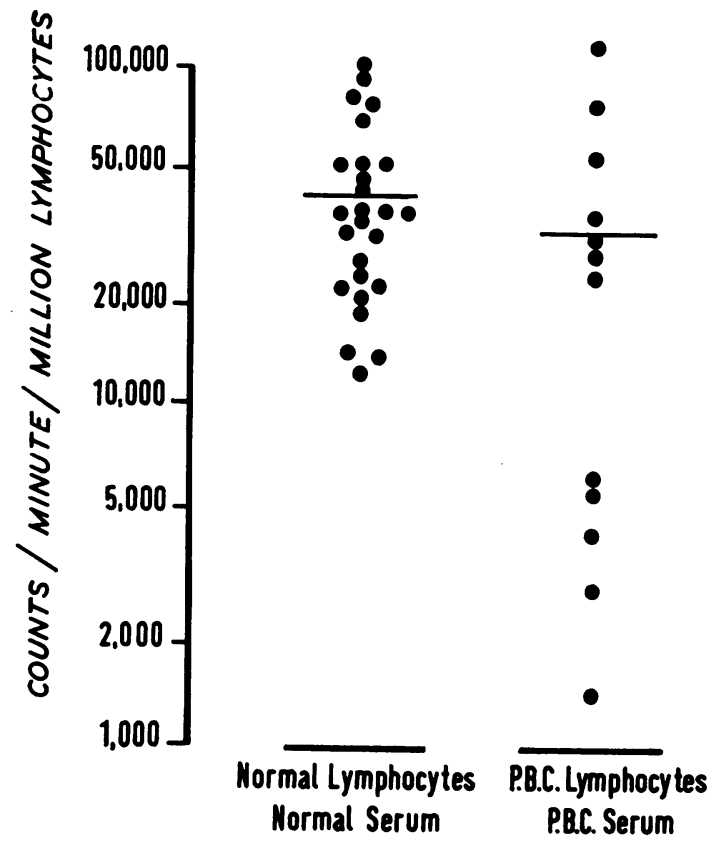

Fig. 1 PHA-induced lymphocyte transformation of normals and patients with primary biliary cirrhosis when performed in the presence of autologous plasma.

culturing normal lymphocytes in normal homologous instead of autologous plasma. The transformation ratio, representing the transformation of lymphocytes in homologous plasma divided by the transformation in autologous plasma, varied from 0.53 to 3.74 . Ratios below or above this range were thus considered to represent an inhibitory or stimulatory effect respectively produced by the use of homologous instead of autologous plasma in the lymphocyte culture system.

The effect of plasma from the 12 patients with primary biliary cirrhosis on the transformation of lymphocytes from 12 normal healthy subjects is also seen in Figure 2. Evidence of an inhibitory factor was found in the plasma of four of the patients. In three the transformation of normal lymphocytes in the presence of primary biliary cirrhotic plasma was below that of our normal control range and in one other the transformation ratio (as defined above) was 0.31 revealing a greater inhibitory effect of the patients plasma than can be expected with normal plasma.

Five of the patients with primary biliary cirrhosis had impaired transformation in autologous plasma. The results of transformation after washing and resuspension in normal plasma are seen in Figure 3.

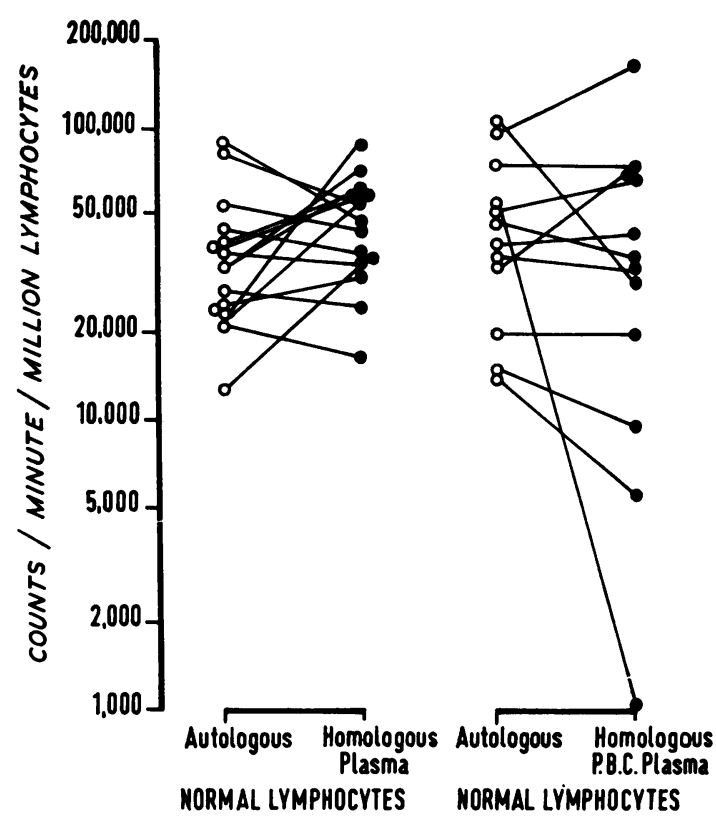

Fig. 2 The effect of homologous normal and primary biliary cirrhotic plasma (৩) on the PHA-induced transformation of normal lymphocytes compared to the response in autologous plasma $(\mathrm{O})$.

In all five patients who had impaired transformation in the presence of their own plasma there was an increase in the uptake of tritiated thymidine when their lymphocytes were cultured in normal plasma. However, in only one instance did the transformation return to within the normal range. In the other four patients with impaired transformation, the transformation ratios (as defined above) were 3.67, $1.67,1.48$, and 3.86 . Only the ratio 3.86 is greater than our established normal range. It can be concluded from these results that of the five patients with impaired transformation there was evidence for a plasma inhibitory factor whose effect could be overcome by washing the lymphocytes and resuspending in normal plasma in only two.

When the primary biliary cirrhotic patients are considered as a group the PHA-induced response of their lymphocytes in the presence of normal plasma and the effect of their plasma on the response of normal lymphocytes is not significantly different from controls. However, it is logical to look only for a plasma inhibitory factor in those patients with impaired transformation, and these results are summarized in Figure 4. In this figure the effect of plasma from the five patients with impaired transformation on the lymphocytes from five normal 


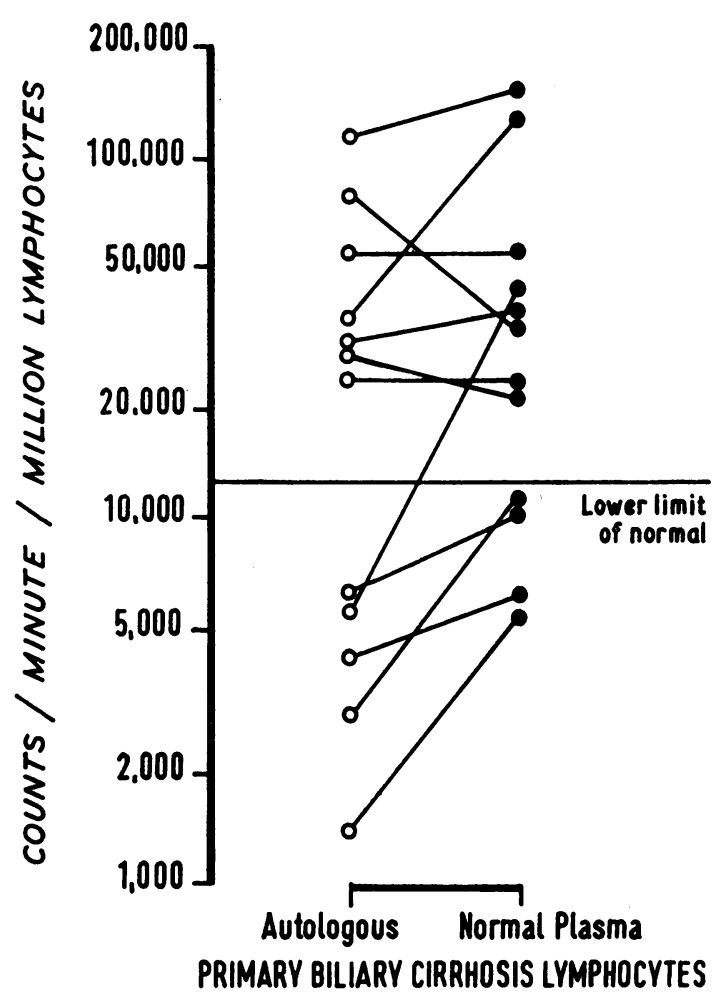

Fig. 3 PHA-induced transformation of lymphocytes from patients with primary biliary cirrhosis cultured in the presence of autologous $(\bigcirc)$ and normal homologous (O) plasma.

individuals can be seen. In four instances the transformation was inhibited but was outside our normal range in only two. If one looks at the transformation of the lymphocytes from the patients, all show improvement in normal plasma, and these results have already been summarized. However, there is not a good correlation between the effect of primary biliary cirrhotic plasma on the transformation of normal lymphocytes and the improvement in transformation following removal of this plasma from the patients' lymphocytes.

\section{Discussion}

It is quite clear from the results of the experiments in normal individuals that washing the lymphocytes and resuspending in plasma from another normal individual results in a slight change in the lymphocyte response to PHA. However, the response remains within the normal range. To demonstrate a significant change in transformation, it must be

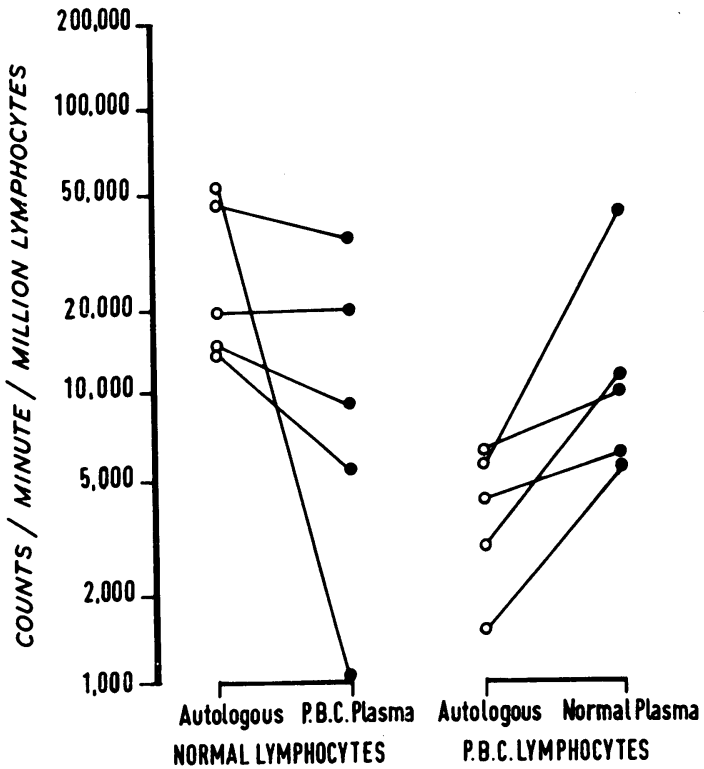

Fig. 4 Role of plasma in producing the impaired $P H A$-induced lymphocyte transformation seen in five patients with primary biliary cirrhosis

quite marked. It can be concluded from our experiments that there is evidence of a plasma inhibitory factor in some patients with primary biliary cirrhosis but that it is unlikely to account completely for the impaired PHA-induced lymphocyte transformation. It is probable that other factors are importanteither deficient T-lymphocytes or an altered population of lymphocytes.

It is unlikely that the washing of the lymphocytes was insufficient to remove all of the plasma inhibitory factors. The work of Hsu and Leevy (1971), Winter et al (1967), and Levene et al (1969) show that these factors when present can be removed by washing. The concentration of plasma used here was about $20 \%$ which should be sufficient to demonstrate the presence of an inhibitory factor. Hsu and Leevy (1971) were able to demonstrate the presence of an inhibitory factor when the concentration of the plasma from their alcoholic cirrhotic patients was only $5 \%$. The results reported here differ from those of Hsu and Leevy, as in their cirrhotics impaired transformation could be completely attributed to the presence of plasma factors. It remains a possibility that there are additional inhibitory factors in the plasma of these patients with primary biliary cirrhosis which have not been demonstrated because of the dosage of PHA we have used. Hsu and Leevy could overcome the inhibitory effect of plasma by increasing the dosage of PHA, and the 
dosage used in our experiments would have been sufficient to overcome the inhibitory effect of plasma in their experiments.

The finding of a plasma inhibitory factor in some patients with primary biliary cirrhosis is of great interest. It has been found that more than $80 \%$ of patients with primary biliary cirrhosis have marked elevation of serum IgM (Feizi, 1968). Morse (1968) has shown that precipitation of PHA occurs with normal human serum, the main serum components involved being $\alpha_{2}$-macroglobulin, $\beta$ lipoprotein, and IgM immunoglobulin. This may be a factor in patients with primary biliary cirrhosis. However, the demonstration of a plasma inhibitory factor in only a few patients and the lack of correlation between impaired transformation and serum IgM concentration (Fox, 1971) makes this unlikely.

These results confirm that a proportion of patients with primary biliary cirrhosis are anergic. The impaired lymphocyte transformation to PHA in vitro can be partially accounted for by the presence of inhibitory factors in the plasma. These are unlikely to account completely for the impaired transformation and it is probable that there are also abnormalities of the T-lymphocytes themselves. F.J.D. was supported by a grant from the Postgraduate Committee of Medicine, University of Sydney.

References

Crowther, D., Fairley, G. H., and Sewell, R. L. (1969). Significance of the changes in the circulating lymphoid cells in Hodgkins' disease. Brit. med. J., 2, 473-477.

Feizi, T. (1968). Immunoglobulins in chronic liver disease. Gut, 9, 193-198.

Fox, R. A. (1971). An immunological study of primary biliary cirrhosis. MD Thesis, University of Newcastle upon Tyne.

Fox, R. A., James, D. G., Scheuer, P. J., Sharma, O., and Sherlock, S. (1969). Impaired delayed hypersensitivity in primary biliary cirrhosis. Lancet, 1, 959-962.

Hsu, C. C. S., and Leevy, C. M. (1971). Inhibition of PHA stimulated lymphocyte transformation by plasma from patients with advanced alcoholic cirrhosis. Clin. exp Immunol., 8, 749-760.

Levene, G. M., Turk, J. L., Wright, D. J. M., and Grimble, A. G. S. (1969). Reduced lymphocyte transformation due to a plasma factor in patients with active syphilis. Lancet, 2, 246-247.

Morse, J. H. (1968). Immunological studies of phytohaemagglutinin. I. Reaction between phytohaemagglutinin and normal sera. Immunology, 14, 713-724.

Winter, G. C. B., McCarthy, C. F., Read, A. E., and Yoffey, J. M. (1967). Development of macrophages in phytohaemagglutinin cultures of blood from patients with idiopathic steatorrhoea and with cirrhosis. Brit. J. exp. Path., 48, 66-80. 